\title{
Developing an Indigenous Cultural Values Based Emoji Messaging System: A Socio-Technical Systems Innovation Approach
}

\author{
Chu Hiang Goh \\ Department of Graphic Communication, \\ School of The Arts, \\ Universiti Sains Malaysia, \\ Penang, Malaysia \\ goh@usm.my
}

\author{
Narayanan Kulathuramaiyer \\ Institute of Social Informatics and Technological \\ Innovations, \\ Universiti Malaysia Sarawak, \\ Kota Samarahan, Malaysia \\ nara@unimas.my
}

\begin{abstract}
Digital divide remains a major concern among indigenous people despite the rapid increase in mobile phone penetration. Beyond the appalling participatory gaps, the devaluing of humanity in the digital era is seen as a much bigger threat. This paper addresses the emerging challenges that are putting indigenous communities at a great disadvantage. The use of emoji in social media and its prominence as a visual language for delivering emotional expressions, gestures and action in a speedy manner is fast becoming a way of life. The universality of emoji poses a major problem to the sustainability of the indigenous cultural values as it imposes western cultural and social behaviour hegemony on the indigenous society. Interactions with remote rural communities in Borneo over the past twenty years have revealed the devastating effect that such an outside-in communication medium has on their cultural resilience. Diversifying the emoji design in adopting a local cultural flavour alone, as described in current literature, will not address the issue effectively. Without considering the whole socio-technical system at a macro-level, the dangers relating to the aggravated disconnect to their rich traditional way of life cannot be averted. We posit a holistic socio-technical systems innovation approach with the participatory involvement of the indigenous community. In this paper, we demonstrate the modeling of complex systems that embed the socio-cultural context without compromising cultural values.
\end{abstract}

\section{CCS CONCEPTS}

- Human-centered computing; • Interaction design; • Systems and tools for interaction design;

\section{KEYWORDS}

Emoji, Co-Design, Socio-Technical Innovation System, Indigenous Communication System

Publication rights licensed to ACM. ACM acknowledges that this contribution was authored or co-authored by an employee, contractor or affiliate of a national govern ment. As such, the Government retains a nonexclusive, royalty-free right to publish or reproduce this article, or to allow others to do so, for Government purposes only. WebSci '20 Companion, July 6-10, 2020, Southampton, United Kingdom (c) 2020 Copyright held by the owner/author(s). Publication rights licensed to ACM. ACM ISBN 978-1-4503-7994-6/20/07 . \$ \$15.00

https://doi.org/10.1145/3394332.3402826

\section{ACM Reference Format:}

Chu Hiang Goh and Narayanan Kulathuramaiyer. 2020. Developing an Indigenous Cultural Values Based Emoji Messaging System: A Socio-Technical Systems Innovation Approach. In 12th ACM Conference on Web Science (WebSci '20 Companion), Fuly 6-10, 2020, Southampton, United Kingdom. ACM, New York, NY, USA, 5 pages. https://doi.org/10.1145/3394332.3402826

\section{INTRODUCTION}

The use of instant messaging is now pervasively covering all aspects of our daily life. Emoji has become the prominent visual language in delivering emotional expression, gesture and action in a speedy nonverbal and non-text endeavour. Emoji are capable of avoiding misunderstanding of the text message content while directing attention to optimum and improved communication among speakers. However, the use of the universal pictograph standard, designed using Unicode, posts problems of ambiguity when it comes to users from different geography, language and cultural backgrounds. As a result, people from a distinct cultural background may interpret the same emoji differently and use it differently in contrast to the original meaning of its design.

\section{THE CHALLENGES OF EMOJI USE IN SOCIAL MEDIA}

In recent years, the use of mobile phones and instant messaging service (IMS) within indigenous community has been on the increase. The challenges posed by social media to the sustainability of the indigenous cultural identity can be described along the following multi-dimensional perspectives:

\subsection{Ambiguity}

The problem of ambiguity in using the standard Unicode emoji is getting more attention due to the isolation of the indigenous community from the outside world, on top of the varied backgrounds within the community. The visual cue provided in the standard emoji looks promising due to its nonverbal and non- text nature. Yet, it can be at times incompatible owing to the missing gesture, human action and emotional expressions according to specific cultural intent. As discovered by Ekman [[5]] from his famous study with the Papua New Guinea indigenous group who were never exposed to the modern culture, some facial expressions are universal, while there are other emotions that are culturally determined. His findings showed that: Fear, Disgust, Anger, Sadness, Enjoyment are universal emotions, while pride, guilt and respect, are mainly 